\title{
Analyse morphosyntaxique de l'article partitif pluriel des en français parlé en république du Congo
}

\author{
Edouard Ngamountsika \\ Université Marien Ngouabi \\ ngamountsika@hotmail.com
}

\section{Introduction}

Dans la pratique du français, la particule de pose le problème de son identification entre l'article partitif et la préposition. En effet, c'est le problème de l'article lui-même car linguistes et grammairiens divergent dans leur traitement de ses formes. Certains considèrent la forme des comme le pluriel de l'article partitif, tandis que d'autres (Charaudeau 1992, Riegel, et alii 1994) la comptent parmi les formes de l'article indéfini. D'autres encore (Grevisse 1986) présentent la forme des comme une forme commune à l'indéfini et à l'article partitif. En dehors de ce flou terminologique, nous constatons l'hésitation dans l'usage de la forme de/des $^{l}$, dans les énoncés négatifs, après certains adverbes de quantité, et quand le SN contient un adjectif antéposé au pluriel. Au lieu d'un de une certaine pratique grammaticale lui préfère des comme dans les exemples du genre : Je n'ai pas des nouvelles de mes parents; beaucoup des enfants sont admis; en tout cas ce sont des grands villageois. Ainsi, l'article partitif se manifeste comme un véritable caméléon aussi bien en ce qui concerne ses formes que pour sa description dans les grammaires $^{2}$. Cet article s'intéressera particulièrement à des emplois qui s'écartent de la norme, c'est-àdire des énoncés où le partitif des s'emploie à la place de. Notre objectif majeur est d'affirmer ou d'infirmer le point de vue de Suzanne Lafage (1985:256) selon lequel les étrangers notamment les Africains ne maîtrisent pas l'usage plénier des déterminants. Nous tenterons de justifier cette hypothèse en nous appuyant sur les grammaires françaises ${ }^{3}$ et sur les études sur la détermination nominale en français parlé africain (désormais FPA). Après une brève présentation théorique sur l'article partitif, notre étude portera, dans l'emploi de l'article partitif dans les énoncés négatifs, ensuite dans sa construction avec l'adverbe beaucoup et en fin dans le schéma DE +adjectif + substantifs au pluriel. Dans un deuxième temps, nous analyserons la façon dont l'article partitif de se substitue à des dans un but purement syntaxique. D’utiles comparaisons avec d'autres aires géographiques seront faites.

\section{Présentation du corpus}

Pour mener à bien notre recherche, nous avons privilégié la linguistique de corpus en nous appuyant sur les travaux de Claire Blanche-Benveniste et du Groupe Aixois de Recherche en Syntaxe (GARS). Nous pensons avec eux que les corpus de langue parlée sont les mieux à même de faire voir ce qui est central et typique dans une langue. Il est nécessaire de travailler sur des enregistrements et des transcriptions authentiques car nous sommes conscients des limites et des faiblesses de l'approche hypothéticodéductive :

Quand on invente des exemples, on confond souvent l'exemple et l'explication, l'exemple étant construit précisément pour justifier et clarifier l'explication. On ne peut pas inventer ce qu'est l'usage ; on peut seulement l'enregistrer. (Sinclair, 1987, cité par Blanche-Benveniste, 1996 : 25)

La question des enregistrements avait déjà attiré l'attention des rédacteurs de L'Élaboration du français élémentaire. Étude sur l'établissement d'un vocabulaire et d'une grammaire de base (1956 : 55)

mais comment faire de la langue parlée une base de dépouillements ? Il va sans dire qu'il est impossible de travailler sur des faits de langue saisis au vol. Il convient donc d'enregistrer par des moyens mécaniques la langue parlée, puis de la transcrire. Les propos, une fois transcrits, sont traités comme les textes écrits des livres ou des lettres. 
L'analyse d'un corpus de français parlé permet donc d'étudier les usages authentiques de la langue française. L'idée de prendre en compte le français parlé pour pouvoir faire une description de la grammaire du français était déjà présente chez Damourette et Pichon. Ils connaissaient la nécessité de s'appuyer sur des exemples attestés : on ne peut pas se permettre d'inventer ${ }^{4}$ les exemples de langue parlée en se fiant à l'intuition, qui est souvent trompeuse.

Nous nous intéressons à un corpus oral établi sans questionnaire préétabli. Quand il s'agit d'études grammaticales, il faut avoir recours à « un corpus ouvert, sans situation d'enregistrement prédéterminé et sans limitation préalable du nombre de locuteurs enregistrés».

Le choix du corpus ouvert n'impliquait pas de parcourir tout le Congo. Nous nous sommes limité à la ville de Brazzaville, centre cosmopolite où coexistent les diverses variétés. Nous n'avons pas non plus sillonné tous les arrondissements de la capitale.

Nous travaillons sur un échantillon varié de situations et des « genres » de prise de parole, de différents types d'évènements de communication, en particulier les monologues, les dialogues et les conversations. Ce qui n'est pas le cas dans la constitution des particularités lexicales en français au Congo. Queffélec et Niangouna (1990:46) reconnaissent que

dans la pratique, l'essentiel de l'enquête a été menée de manière moins rigoureuse : les chercheurs ont utilisé des méthodes moins sophistiquées. Ils ont fait appel à la technique du questionnaire préétabli ou demandé à des interlocuteurs complaisants d'évoquer dans des conversations plus ou moins dirigées tel ou tel thème de la vie courante.

Contrairement à ces chercheurs, notre enquête ne comportait aucune directive à l'endroit des interlocuteurs. Les locuteurs étaient libres d'aborder des sujets de leur choix.

Notre corpus transcrit comporte 56.668 mots et repose sur les enregistrements effectués de 2003 à 2004 à Brazzaville. Il est constitué de douze (12) textes. Ces textes comportent un monologue, et des dialogues réunissant deux, trois, cinq et six locuteurs engagés dans une interaction.

Notre population cible est composée de locuteurs ayant un niveau d'études allant du Certificat d'études primaire et élémentaire (CEPE) à la licence et qui utilisent ce que nous appellerons par commodité « la parlure congolaise» par opposition à ce qui serait le français des intellectuels. Nous remarquons que les locuteurs ayant un niveau scolaire entre le CEPE et le Brevet d'études du premier cycle (BEPC) produisent tantôt des énoncés standard, tantôt des énoncés non standard. Cette observation est aussi valable pour les étudiants. Ce qui, de toute évidence, met en doute les critères de scolarisation pour justifier les énoncés hétérogènes attestés. Mais cela suffit-il pour dire que les scolarisés sont influencés par la norme endogène? Ou sont-ils victimes de l'hypercorrection? S'ils évitent d'utiliser le "gros français », ce n'est pas par incompétence mais pour ne pas être coupé de la masse congolaise. L'objectif n'est pas toujours de montrer une prouesse oratoire mais de transmettre un message, ce que Henri Frei qualifie sans doute de «besoin de brièveté» et de « besoin d'expressivité». On retrouve la loi bien connue du moindre effort. Il s'agit soit d'aller plus vite, soit d'articuler avec le maximum d'économie. La raison qui motive les sélections des articles partitifs sont dues à la singularité d'abord des énoncés relevés dans notre corpus mais aussi parce que nous avons retrouvé les mêmes constructions «malmenées » depuis le XVII e siècle. Nous avons estimé qu'il serait nécessaire d'aborder cette épineuse question de l'article en français.

\section{De l'emploi de l'article partitif}

Selon Riegel et alii (1994: 161), l'article partitif s'emploie « devant le singulier des noms de masse ( $d u$ plâtre, de la farine) et des noms dits" abstraits " qui ne renvoient pas à des entités comptables $(d u$ courage, de la lâcheté) ». or la plupart des exemples fournis dans un certain nombre de grammaires françaises (Boularès \& Frérot 1997, de Salins 1996, Grevisse 1986, Judge \& Healey 1985, Pedersen, Spang-Hanssen \& Vikner, 1980, Riegel,Pellat\& Rioul, 1994, Wagner \& Pinchon 1962), on constate que les premiers exemples parlent d'habitude de vin, de bière, de pain, de soupe, etc. Si les grammaires 
mentionnent, en deuxième lieu, des sentiments comme du courage, de la patience, il est plus rare de trouver des exemples comme de la chance, du travail, du soleil, de la neige, du brouillard (de Salins1996) ${ }^{5}$. Il s'agira pour nous d'étudier le morphème des partitifs dans un corpus du français parlé recueilli à Brazzaville.

\section{Selon la pratique congolaise}

\subsection{Distribution des déterminants en FPC}

Le Français Fondamental a mis en exergue l'intérêt de l'analyse par fréquences en vue de donner une meilleure représentation de la langue parlée. Il est donc important d'étudier la fréquence des divers déterminants en FPC.

\begin{tabular}{|l|c|c|}
\hline Déterminant & Réels & $\%$ \\
\hline Article défini & 2808 & 56,61 \\
\hline Article indéfini & 781 & 15,74 \\
\hline Article partitif & 125 & 2,52 \\
\hline Quantifieurs & 249 & 5,02 \\
\hline Démonstratif & 534 & 10,76 \\
\hline Possessif & 463 & 9,33 \\
\hline Total & 4.960 & 100 \\
\hline
\end{tabular}

Tableau 1 distributionnels des déterminants

En étudiant l'ensemble des déterminants, on constate que le français parlé congolais utilise davantage les articles définis. Les locuteurs congolais ont plus utilisé l'article défini (56,61\%). En second rang l'article indéfini est moins représenté qu'en FPC (15,74 \%); l'article partitif est rarement utilisé en FPC (2,52\%); les déterminants quantifieurs sont en revanche moins attestés $(5,02 \%)$; le démonstratif est assez fréquent en FPC $(10,76 \%)$. Le FPC $(9,33 \%)$.Ces données nous permettent d'affirmer d'une façon générale qu'en français parlé africain, les locuteurs préfèrent les déterminants définis aux indéfinis. Ainsi se trouvent confirmés le suremploi des définis, le sous-emploi des indéfinis et la rareté des partitifs. Le suremploi des définis apparaît naturel dans la mesure où les déterminants définis « ont en commun de présupposer que le référent du nom est identifiable par le locuteur (...) les indéfinis présentent le référent du nom non identifiable par l'interlocuteur. » (Leeman, $2004: 44,45$ )

\subsection{L'article en question}

En raison de son absence en langue latine et de l'hésitation de certains grammairiens à le reconnaître comme partie du discours, l'article était et reste l'une des questions délicates de la syntaxe du français. Or «Une conséquence de l'emploi de l'article, c'est que les attributs de fonction, de nombre et de genre déjà assignés au nom sont de nouveau assignés à l'article, nom de l'extensité. Il y a accord du nom et de l'article en fonction, en nombre et en genre avec le nom.» pense Guillaume (2003: 136). Comme 
l'illustre ce tableau, nous nous limiterons à l'article partitif qui est le moins utilisé. Le système de base des quantifiants est constitué par les articles un et le. Nous fonderons la description des quantifiants sur la psychomécanique du langage qui postule le cinétisme de la signification. En l'espèce, cela veut dire que le signifié de langue de un n'est autre qu'une tension, « un mouvement de pensée portant la notion substantivale en direction d'une image particulière », tandis que celui de le correspond à une seconde tension, «un mouvement de pensée portant le substantif à une image générale de lui-même » (1981 : 132). Les quantifiants bipolaires se résument dans le tenseur binaire développé par Moignet. En représentation :

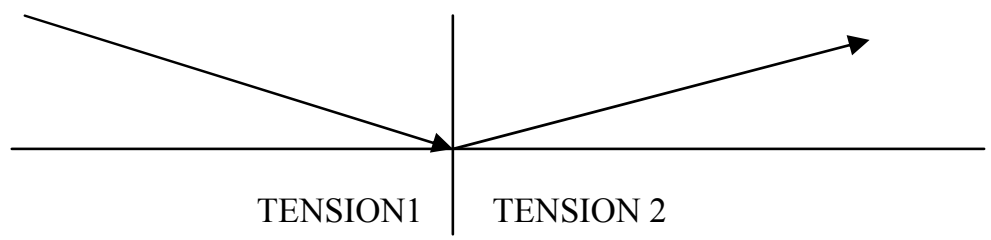

(mouvement de particularisation) (mouvement de généralisation)

UN LE

Le tenseur binaire, Moignet (1981: 132)

Il est reconnu de longue date que l'article français comporte deux éléments, traditionnellement appelés « article indéfini» et «article défini» auxquels s'ajoutent un «article partitif» et «l'article zéro ». En représentation :

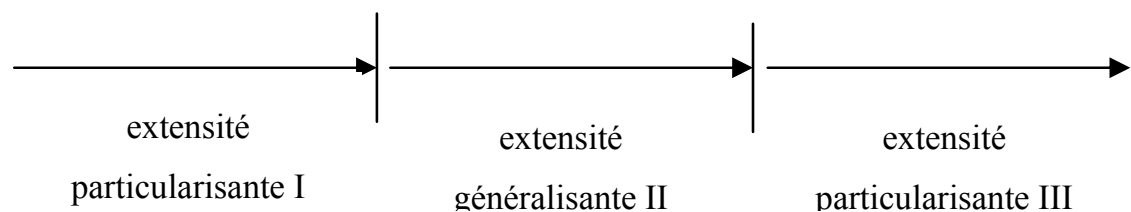

Le tenseur à trois tensions

De ces trois extensités, il faut ajouter « l'article zéro qui est le signe du non-engagement du mécanisme de régulation d'extensité » (Soutet, 2005,22). L'ensemble du système des quatre articles se résume ainsi :

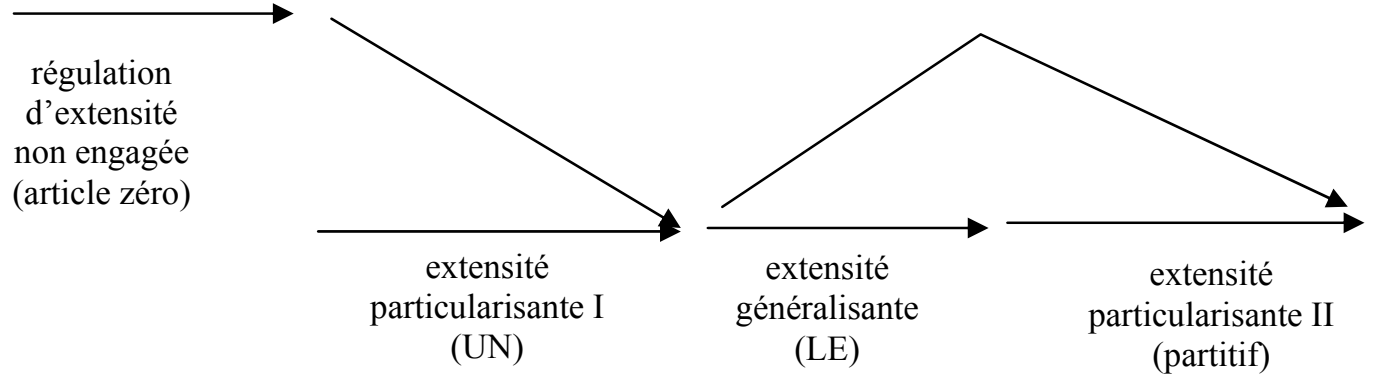


Le tenseur à quatre tensions Soutet $(2005: 23)$

Il s'agit donc ici de mener une étude détaillée de ces différents articles à la lumière de ce schéma. Nous commencerons d'abord par montrer des exemples standard en insistant sur des emplois restructurés en FPC.

\subsection{Article partitif en FPC}

Une littérature abondante existe sur l'origine, la nature et la fonction du morphème de dans le partitif. Les spécialistes se divisent en deux camps : les tenants de l'analyse comme préposition et les partisans de son interprétation comme déterminant ou prédéterminant nominal (Wilmet $1986: 112$ ). Nous commenterons uniquement les énoncés en FPC dans lequel de est déterminant. Toutefois, nous nous inspirons particulièrement d'Englebert (1992) pour illustrer nos analyses dans une démarche de type guillaumien.

Le déterminant partitif est d'un emploi aussi rare en FPC (2,56 \%). La différence n'est pas trop grande. En dehors des emplois standard du partitif en FPC, trois problèmes majeurs se posent : le remplacement du partitif par l'indéfini, son emploi dans les phrases négatives et la confusion des/de après le quantifiant positif beaucoup.

\subsection{La confusion dans l'emploi de l'article défini pour le partitif}

Notre corpus présente des occurrences où le déterminant défini «s'emploie (...) pour marquer la valeur générique des noms dits massifs (le vin/la farine/le courage/la tendresse) dont les occurrences particulières sont construites au moyen de l'article partitif» (Riegel et alii, ibid.,161). Il s'agit pour la plupart de temps des noms massifs par exemple des noms de matières «à référence homogène (vin, marbre, oxygène » Flaux, 1997, 37). Tel est le cas de l'exemple 1 où au lieu d'utiliser une extensité particularisante II $d u$ développée par Olivier Soutet, nous avons une exensité généralisante le.

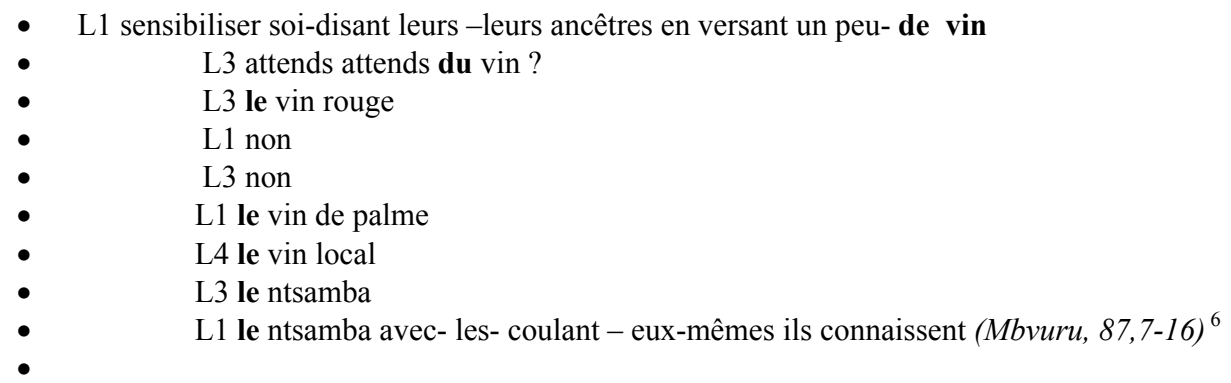

Pour cerner les différentes nuances de l'emploi générique de l'article défini, nous avons recouru à un critère de «substitution dans la catégorie des adjectifs déterminatifs; il faut recourir à des déterminants adverbiaux (Du vin, j'en veux un peu) ou nominaux (Du vin, j'en veux un verre)» (Flaux, ibid., 36). Lorsque nous appliquons cette méthode à certains de nos énoncés, nous nous retrouvons avec des emplois non standard de l'article défini :

- $\quad$ il vit comment comment comment elle vit- il / fait, faut/ le courage il faut avoir seulement le courage c'est tout-- (C, 39, 15-12)

- L1 moi la bière tu sais : c'est+ moi la bière : ça me donne le courage

- $\quad$ L2 mais le courage mais : tu vois c'est ça hein + tu perds (AS, 192, 10-11)

\subsection{Des dans les énoncés négatifs}

Un autre aspect caractéristique du FPC est l'emploi du morphème des dans les énoncés négatifs .

- quand il étai ministre-là - là il euh ne multipliait pas des descentes à Makazou (C., 12,45)

- mais quand il était ministre- il ne s'occupait pas des gens de Makazou (C.,17,45) 
En effet, selon Muller, (cité par Leeman 2004 : 147), «lorsque la phrase est négative, le sens est nécessairement partitif». Ces phrases ne contiendraient pas en principe le partitif pluriel des. Comme le soulignent Wagner et Pinchon (1962: 97) que « dans les phrases négatives qui ont un caractère absolu, c'est la préposition $d e^{7}$ qui précède un substantif au pluriel ou un substantif au singulier ayant une valeur collective ». Grevisse (1980§664) abonde dans le même sens lorsqu'il affirme: « devant un objet direct ou sujet " réel " dans les phrases négatives, on emploie le simple de servant d'article partitif ou indéfini, si la négation est absolue, c'est-à-dire si le nom peut être précédé de "aucun " ou de " aucune quantité de" ».

Dans l'exemple 4, nous aurons un de et non des. Il s'agit d'une négation absolue dont parle Grévisse. En dehors du test de substitution du partitif des par aucune, l'incidence interne du verbe multiplier proscrit l'usage d'un des. La présence d'un des est encore présente dans ces énoncés où les locuteurs estiment que dire de serait une faute.

- aux petits enfants - à ceux qui n'ont pas des armes- (Mbvuru, 90,13)

Or le même phénomène est aussi constaté par Biloa pour le Cameroun (1998) :

- Je n'ai pas des parents à Maroua

Et par Gaadi pour le Maroc (1998) :

- Je ne vois pas des exemples.

L'emploi de/des dans les énoncés négatifs s'explique selon Englebert (1996:24-25) par le fait que " l'emploi de tient à sa corrélation nécessaire avec un quantifiant, ici un quantifiant périphérique. » Cette concurrence entre de et des est encore récurrente en français écrit chez certains étudiants à l'université Marien Ngouabi où dans le cadre d'un cours de technique d'expression française dans certains départements de la faculté des lettres et des sciences humaines, les étudiants ne choisissent pas convenablement le morphème de ou des. L'exercice consistait à nier les phrases suivantes nous avons du manioc à la maison; je bois du vin. La tendance est souvent de substituer un des au lieu de de. Or, c'est un fait connu au XII ${ }^{\mathrm{e}}$ siècle qu'en phrase négative dans un $\mathrm{SN}$ objet direct, la forme des prend dans la variété standard la forme $d e$. Selon Englebert (1992: 147), en effet « c'est un fait connu et reconnu que la langue du XII ${ }^{\mathrm{e}}$ siècle garde encore le souvenir de l'origine substantivale de ces " auxiliaires [pas, point, mie] ", de sorte que certains considèrent que le DE dont ils peuvent se faire suivre n'introduit rien d'autre qu'un complément du nom.» La restructuration du français est encore perceptible avec l'usage de l'adverbe de quantité beaucoup.

\subsection{Avec l'adverbe de quantité beaucoup + des}

Un autre aspect ambigu en FPC est la construction syntaxique beaucoup + Des. Elle est influencée par le substrat linguistique congolaise où l'adverbe miingi ou ébélé se traduirait en français par beaucoup, assez, nombreux. Le passage de l'idéogenèse à la morphogenèse pour parler comme les guillaumiens influencent leur parlure.

- $\quad$ oh même pas couiller les femmes il y a beaucoup des affaires que tu peux fait (C., 39,3)

- c'est lui qui va faire revenir beaucoup des étudiants à la deuxième session (DC, 161,19-20)

- après avoir recruté beaucoup des africains dans leur équipe et à croire euh

- il a beaucoup volé et il a beaucoup acheté des parcelles

- il a acheté au moins des parcelles

Et pourtant Grevisse (1993: 872) souligne que «quand les adverbes de degré assez, beaucoup, combien, moins, plus, trop, etc., sont employés comme déterminants indéfinis, c'est par de qu'ils sont unis au nom dans le français régulier» (C'est l'auteur qui souligne). Aussi constate-il que «la langue populaire de diverses régions, surtout avec beaucoup, emploie la forme pleine de l'article ». Nous ne pensons pas, par ailleurs, que dans les exemples 9, 10, 11 et 12, l'emploi du partitif pluriel s'agisse de la langue populaire congolaise. Il est très tôt de parler d'un français populaire congolais. Il faudrait plusieurs études pour arriver à cette conclusion. D'ailleurs ces propos sont tenus par des étudiants ayant validé leur 
licence es lettres. La syntaxe de beaucoup en 13 est très orale dans la mesure où l'adverbe de quantité est placé entre l'auxiliaire et le participe passé. Le locuteur insiste ici sur la quantité. En 15 par exemple, le locuteur aurait utilisé un de au lieu de de l'.

- le commerce de poisson il faut avoir beaucoup de l'argent-

La construction beaucoup + des est aussi attestée au Maroc puisque Driss Gaadi $(1998: 232)$ cite des exemples tels :

- On voit beaucoup des jeunes qui font la broderie

- A Rabat on trouve pas mal des coopératives, beaucoup des associations...

Quoique que Grevisse (1993) et Wilmet (2003) reconnaissent que l'emploi de l'adverbe beaucoup et de l'article partitif des soit une propriété du français populaire, cette construction demeure d'un usage délicat. S'il est facile d'expliquer son emploi en français parlé, il faudrait reconnaitre qu'il est d'un emploi ambigu en français tout court. La sémantèse de beaucoup serait à l'origine de son adjonction au pluriel des. Les locuteurs pensent peut-être qu'il faudrait joindre à ce quantifiant un morphème pluriel des et non $d e$. Nous assistons en quelque sorte à une simplification interne de la langue. Dans les énoncés négatifs en discours par contre, cela révèle le caractère difficile et ambigu du morphème de en français parlé.

\subsection{Dans les énoncés DES + adjectif+ substantif au pluriel}

Fournier (2002 : 162) rapporte que Maupas avait déjà consigné l'alternance de/des dans le syntagme DE+ Adjectif + substantif au pluriel avant que Vaugelas ne l'édicte comme « une règle essentielle dans la langue », ce qu'on est en droit d'appeler la fameuse règle de Maupas. Cette loi consiste à ce que «des "s'allège" en de lorsqu'il introduit un nom précédé de l'adjectif» souligne Leeman (2004:10). Du français classique à nos jours, cette règle souffre malheureuse d'entorse. Fournier constate déjà qu'au XVIIe siècle, la règle est « loin cependant d'être d'une application constante» :

- Il donna des sensibles marques de la peur (Molière, P.El,II)

- Elle est donc, de celles que l'on appelle des éternelles vérités (La Bruyère, Car. XVI, 47)

Cette entorse de la règle de Maupas est aussi constatée en FPC où les locuteurs antéposent un des devant l'adjectif épithète :

- on dit que les les chargeurs ce sont des grands voleurs ( M., 45,9)

- je connais des petites choses (RSQ., 19,29)

La loi de Maupas est aussi malmenée notamment au Sénégal où Moussa Daff (1998: 109) relève :

- Bon même au 19e siècle la religion a été fustigée par euh des grands théoriciens

- (...) qui sont sur le marché de la concurrence qui est très rude bon ils trouvent souvent sur ce terrain là des vieux loups de mer voyez qui sont sans pitié

Ou Gaadi pour la Tunisie :

- On lui dit des fausses choses.

- El Maâri et Ibn Rochd étaient des grands poètes qui voient.

Edmond Biloa note la même récurrence en français parlé du Cameroun :

- Ma sœur ne m'a pas acheté des beaux vêtements au Bénin.

Constaté et tancé depuis près de quatre siècles par des grammairiens (Maupas, Vaugelas), l'usage de l'article partitif dans le syntagme DES + Adjectif + substantif au pluriel est toujours d'actualité. $\mathrm{Au}$ regard de ces trois inverseurs qui n'appellent pas des une extensité particularisante I qu'une extensité II. Nous avons l'impression que le substantif est doublement actualisé. 


\section{Essai d'explication}

\subsection{Dégradation de l'usage}

Pour les pays africains la première explication de la syntaxe malmenée de l'article partitif $p$ s'expliquerait par la carence éducative. L'enseignement des déterminants est très délicat pour les jeunes maitres dont la formation est parfois approximative. Mais aussi du crédit souvent accordé au cours de grammaire française «discipline scolaire, faite de définitions obscures, de règles suivies d'une multitude d'exceptions incompréhensibles » comme le souligne Muller (2002:11). Que dire en ce moment là des occurrences relevées en français de l'Hexagone. Comment expliquer aux apprenants congolais par exemple que le morphème des peut être tantôt article partitif pluriel tantôt article indéfini. Ce caractère caméléon du morphème des relèverait à notre avis de la complexité du français.

\subsection{L'influence du français}

Les constructions malmenées en FPC sont essentiellement intrasystémiques. Elles sont inhérentes à la langue française elle-même, c'est-à-dire dans son système. En effet, en français classique déjà, Fournier (2002 : 164) note la concurrence de/des en contexte négatif alors que la construction était fustigée par Vaugelas. Elle relève des occurrences suivantes :

- $\quad$ Madame, je n'ai point des sentiments si bas. (Racine, Phèdre, 595)

- Je ne vous ferai point des reproches frivoles (Baj., 1470)

- Il n'avait pas des ${ }^{8}$ outils à revendre. / Sur celui-ci roulait tout son amour. (La Fontaine, Fables, V, 1)

Les linguistes devraient par exemple trancher sur le bon emploi. Cinq siècles après Vaugelas la construction demeure. En ce moment-là, nous ne pouvons pas partager le point de vue de certains linguistes qui pensent que les écarts rencontrés en FPC sont le fruit du français populaire africain. Il s'agit du français tout court où «dans l'homme pensant, l'existence d'un univers - idée expansif, voué à l'accroissement par quantité et par qualité, univers intérieur qu'il est seul, parmi les êtres pensants, d'édifier lui-même » Guillaume (2003 : 136). C'est certainement en ce moment là, le moi pensant de l'homme se représente l'univers au pluriel d'où la présence de des. Nous percevons par la difficulté de l'usage de ce morphème. Nous pourrions par exemple esquisser de nouvelles pistes d'enseignements des déterminants notamment le morphème polymorphe des.

\subsection{Esquisse d'une nouvelle didactique}

L'enseignement des déterminants en République du Congo passe sous silence la question de l'article partitif. Il aborde l'enseignement des articles définis et indéfinis d'une manière traditionnelle en se focalisant sur la morphologie. Alors qu'il serait souhaitable d'insister sur le contexte d'emploi mettant en exergue les objets massifs et des entités non comptables. Il faudrait par exemple bien distinguer l'article indéfini pluriel du partitif pluriel.

Et multiplier des astuces pour distinguer un des indéfini, d'un des partitifs. Par exemple ${ }^{9}$ dire aux apprenants que de remplace les partitifs avec des expressions de quantité :

- Je mange cent grammes de viande

- Je bois beaucoup de café

- J'achète deux bouquets de fleurs

Ou encore dire qu'à la forme la négative des devient $d u$.

- Je ne mange pas du fromage

- Je ne bois jamais du thé

Plusieurs approches sont possibles pourvue que la notion soit bien assimilée.Sur le plan linguistique, il y a, peu de différence entre "des" partitif (je mange des rillettes, des confitures...) et "des" indéfini (je 
mange des bonbons, je lis des journaux...), mais il y a une différence plus grande entre "des" indéfini et "des" article contracté "de+les" dans j'ai lu des livres très intéressants / il a déchiré la couverture des livres que tu m'avais prêtés $=$ de+les. C'est pourquoi nous pensons qu'il faudrait justement revoir la terminologie en retenant que la notion d'extensité particularisante. Le test de la négation serait donc important :

je ne mange pas de rillettes // je ne lis pas de journaux

je n'ai pas lu de livres intéressants // il n'a pas déchiré la couverture des livres que tu m'avais prêtés

La négation fait partie de la quantification, puisqu'elle signifie une quantité nulle. Or avec les quantificateurs on a aussi une opération de prélèvement quantitatif, et la substance sur laquelle est opérée le prélèvement est démarquée à l'initiale par la préposition "de"cf. un kilo de rillettes // un peu de rillettes //

\section{Conclusion}

L'article partitif pluriel des à tendance à suppléer de dans les énoncés négatifs, devant le quantificateur beaucoup et devant l'adjectif qualificatif épithète dans la pratique du français en diachronie et en synchronie. Ainsi récusons-nous le point de vue de Lafage $(1985: 256)$ selon lequel « pour les étrangers, l'usage plénier de l'article français est difficile à acquérir compte tenu des diverses nuances qui peuvent être exprimées par sa présence, son absence, son genre, son nombre... ». L'usage plénier de l'article partitif à la lecture de ces différents exemples montre le caractère caméléon de/des en français du XVII ${ }^{\mathrm{e}}$ siècle à nos jours. Cela s'expliquerait par le fait que l'article partitif n'existait pas en ancien français ${ }^{10} \mathrm{ou}$ par sa complexité d'être tantôt article tantôt préposition.

\section{Bibliographie}

Bel-Hadj Larbi, L. (1998). Le français parlé par les intellectuels tunisiens bilingues : une étude morphosyntaxique, in Queffélec, A. [éds] Alternances codiques et français parlé d'Afrique, Aix en Provence, pp.31-37

Blanche-Benveniste, C. (1996). De l'utilité du corpus linguistique, Revue française de linguistique appliquée, I-2, 2542.

Biloa, E. (1998). La syntaxe du français parlé au nord Cameroun. Le Français en Afrique, n 15, pp.

Daff, M. (1998). Petite vitrine syntaxique du français oral au Sénégal, in Queffélec, A. [éds] Alternances codiques et français parlé d'Afrique, Aix en Provence, pp.107-117.

Englebert, A. (1992). Le « petit mot» De. Étude de sémantique historique. Genève:Droz.

Flaux, N. (1997). Les déterminants et le nombre, in Flaux. N.Van De Velde.D.De Mulder.W. [Dir.].Entre général et particulier : les déterminants, Artois : Presses Universitaires, pp.17-82.

Fournier, N. (2002). Grammaire du français classique. Paris: Belin.

Gaadi, D. (1998). De quelques particularités morpho-syntaxiques du français au Maroc, in Queffélec, A. [éds] Alternances codiques et français parlé d'Afrique. Aix en Provence :PUP, pp.229-236.

Gougenheim, G., Rivenc, P., Michea, R. \& Sauvageot, A. ([1956]1964). L'élaboration du français fondamental ( $1^{\text {er }}$ degré) .Étude sur l'établissement d'un vocabulaire et d'une grammaire de base. Paris : Didier.

Guillaume, G. (2003). Prolégomènes à la linguistique structurale 1. Lowe Ronald [éds] Québec, PUL

Henriksen, T. (2001). La mise en scène de l'article partitif. http://www.duo.uio.no/roman/Art/Rf2001.13$1 / 01 \% 20$ Henriksen.pdf

Lafage, S. (1985). Français écrit et parlé en pays éwé (Sud-Togo). Paris, Ministère des Relations extérieures et SELAF (CNRS). Coll. Sociolinguistique $\mathrm{n}^{\circ} 3$.

Leeman, D. (2004). Les déterminants du nom en français : syntaxe et sémantique. Paris : PUF 
Muller, C. (2002). Les bases de la syntaxe. Syntaxe contrastive français-langues voisines. Bordeaux : PUB.

Moignet, G. (1982). Systématique de la langue française. Paris : Klincksieck.

Ngamountsika. É. (2007). Le français parlé en République du Congo : étude morphosyntaxique. Thèse pour le doctorat, NR, Aix en Provence \&Brazzaville.

Queffélec. A.[éds] (1998). Alternances codiques et français parlé en Afrique. Actes du colloque d'Aix-en-Provence, septembre 1995, Aix en Provence : PUP.

Queffélec, A. et Niangouna. A. (1990). Le français au Congo (RPC). Aix en Provence : PUP.

Riegel, M. , Pellat, J-C \& Rioul, R. (1994). Grammaire méthodique du français. Paris : PUF.

Soutet, O. (2005). La syntaxe du français, Paris. PUF « Que sais-je ?»

Togeby, K. (1974). Précis historique de grammaire française. København: Akademisk Forlag.

Wagner, R.-L. \& Pinchon, J. (1962). Grammaire française classique et moderne. Paris : Hachette.

Wilmet, M. (2003). La grammaire critique du français. Louvain-la-Neuve : Hachette Duculot.

Wilmet, M. (1986). La détermination nominale. Paris : PUF.

${ }^{1}$ Ce postulat avec de n'est pas souvent respecté où de se substitue à des. C'est d'ailleurs cet aspect déviant que nous allons analyser.

${ }^{2}$ Turid Henriksen (2001)

${ }^{3}$ Wagner \& Pinchon (1962), Grevisse (1986), Fournier (2002), Leeman (2004), Englebert (1992), Riegel et alii et de Wilmet (1986) et (2003)

4 Malheureusement beaucoup de lexicologues africains et africanistes n'appliquent pas ce principe. Ils forgent au gré des exemples qui sont en réalité le fruit de leur intuition.

${ }^{5} \mathrm{Cf}$. Turid Henriksen

${ }^{6}$ Il convient de signaler que la lettre désigne l'abréviation du titre, le premier chiffre la ligne et le deuxième la page.

${ }^{7}$ Wagner \& Pinchon le qualifie de préposition alors qu'ils traitent de déterminants spécifiques.

${ }^{8}$ Damourette et Pichon (\$386), cités par Nathalie Fournier $(2002: 164)$ commentent ainsi le vers de La Fontaine : Il n’avait pas des outils à revendre, dans lequel la négation porte sur le quantificateur des :

La négation porte ici sur l'article des. -Avoir des outils à revendre, c'est en avoir beaucoup, en avoir en quantité commerciale. Le bûcheron n'en avait pas en pareille quantité; c'est la quantitude qui doit être niée; aussi est-il nécessaire que l'article qui l'exprime soit maintenu auprès du forclusif, puisque c'est sur lui que la valeur sémantique du forclusif doit tomber.

${ }^{9}$ Ces éléments ont été pris sur le net www.polarfle.com/exercice/elempartitif.htm

${ }^{10} \mathrm{C}$ 'est dans l'emploi de la préposition de pour indiquer une partie de ce qu'on boit ou mange qu'on trouve son origine. L'emploi de cet article, tel qu'on le connaît aujourd'hui, s'est généralisé en français au XVIe siècle (Togeby 1974: 47). 